\title{
On quantitative noise stability and influences for discrete and continuous models
}

\author{
RAPHAËL BOUYRIE \\ University of Marne-La-Vallée, France
}

\begin{abstract}
Keller and Kindler recently established a quantitative version of the famous Benjamini Kalai-Schramm Theorem on noise sensitivity of Boolean functions. The result was extended to the continuous Gaussian setting by Keller, Mossel and Sen by means of a Central Limit Theorem argument. In this work, we present an unified approach of these results, both in discrete and continuous settings. The proof relies on semigroup decompositions together with a suitable cut-off argument allowing for the efficient use of the classical hypercontractivity tool behind these results. It extends to further models of interest such as families of log-concave measures and Cayley and Schreier graphs. In particular we obtain a quantitative version of the B-K-S Theorem for the slices of the Boolean cube.
\end{abstract}

MSC: 60C05; 05D40

Keywords: Boolean cube, Schreier graphs, Gaussian measure, Influence, Noise stability, Hypercontractivity.

\section{Introduction}

The notion of influences of variables on Boolean functions has been extensively studied over the last twenty years, with applications in various areas such as combinatorics, statistical physics and theoretical computer science, in particular cryptography and computational lower bounds (see e.g. the survey [K-S]). Similarly, the noise sensitivity of a Boolean function is a measure of how its values are likely to change under a slightly perturbed input. Noise sensitivity became an important concept which is useful in many fields such as for instance percolation, from which it was originally defined in [B-K-S]. For an overview of the topic, see e.g. the book [G-S], in which the noise sensitivity concept and the results of [B-K-S] are presented in the context of percolation theory.

Noise sensitivity and influences are closely related. In this work, we will be concerned with recent connections between influences and asymptotic noise sensitivity. To start with, let us recall these two important concepts on the discrete cube $\{-1,1\}^{n}$. Rather than noise sensitivity, we describe the related notion of noise stability. Throughout this paper, denote by $\nu$ the product measure $\left(p \delta_{-1}+(1-p) \delta_{1}\right)^{\otimes n}$ on $\{-1,1\}^{n}$ where $p \in(0,1)$.

Let $A \subset\{-1,1\}^{n}$. For $x=\left(x_{1}, \ldots, x_{n}\right) \in\{-1,1\}^{n}$ and $i=1, \ldots, n$, let $\tau_{i} x \in\{-1,1\}^{n}$ be the vector obtained from $x$ by changing $x_{i}$ in $-x_{i}$ and leaving the other coordinates unchanged. The influence of the $i$-th coordinate on a function $f:\{-1,1\}^{n} \rightarrow \mathbb{R}$ is given by

$$
I_{i}(f)=\left\|f(x)-f\left(\tau_{i} x\right)\right\|_{L^{1}(\nu)},
$$


Similarly, for sets $A \subset\{-1,1\}^{n}$, the influence of the $i$-th coordinate is defined using characteristic functions by $I_{i}(A)=I_{i}\left(\mathbf{1}_{A}\right)$. Notice then that in the uniform case $(p=1 / 2)$, it holds

$$
I_{i}(A)=2 \nu\left\{x \in\{-1,1\}^{n} ; x \in A, \tau_{i} x \notin A\right\} .
$$

Turning to noise stability, let $\eta \in(0,1)$ and let $X=\left(X_{1}, \ldots, X_{n}\right)$ be distributed according to $\nu$ on $\{-1,1\}^{n}$. Let $X^{\eta}=\left(X_{1}^{\eta}, \ldots, X_{n}^{\eta}\right)$ be a $(1-\eta)$-correlated copy of $X$, that is $X_{j}^{\eta}=X_{j}$ with probability $1-\eta$ and $X_{j}^{\eta}=X_{j}^{\prime}$ with probability $\eta$ where $X^{\prime}$ is an independent copy of $X$. For a function $f:\{-1,1\}^{n} \rightarrow \mathbb{R}$, following the notations of [K-M-S2], define its noise stability as

$$
\mathcal{S}_{\eta}^{c}(f)=\mathbb{E}_{\nu}\left[f(X) f\left(X^{\eta}\right)\right]-\mathbb{E}_{\nu}[f(X)]^{2} .
$$

Similarly, for a subset $B \subset\{-1,1\}^{n}$, define its noise stability by $\mathcal{S}_{\eta}^{c}(B):=\mathcal{S}_{\eta}^{c}\left(\mathbf{1}_{B}\right)$. Thus, the noise stability of a function $\mathcal{S}_{\eta}^{c}(f)$ is a measure of the sensitivity of $f$ to a small noise $\eta$ in its input. A sequence of functions/sets $\left(f_{n_{\ell}}\right)_{n_{\ell}},\left(B_{n_{\ell}}\right)_{n_{\ell}}$ over $\{-1,1\}^{n_{\ell}}, \ell \in \mathbb{N}$, where $n_{\ell} \nearrow \infty$, is said to be (asymptotically) noise sensitive if its noise stability tends to 0 as $\ell$ tends to $\infty$, that is

$$
\lim _{\ell \rightarrow \infty} \mathcal{S}_{\eta}^{c}\left(f_{n_{\ell}}\right)=0
$$

or

$$
\lim _{\ell \rightarrow \infty} \mathcal{S}_{\eta}^{c}\left(B_{n_{\ell}}\right)=0
$$

for each $\eta \in(0,1)$ (keeping in mind $\eta$ small but fixed).

A first connection between noise sensitivity and influences have been established by Benjamini, Kalai and Schramm in the paper [B-K-S]. They gave a criterion for a sequence a sets to be noise sensitive in terms of the sum of squares of the influences. More precisely, one of the main results of B-K-S] is the following theorem.

Theorem 1.1. Let $B_{\ell} \subset\{-1,1\}^{n_{\ell}}, \ell \in \mathbb{N}$. If

$$
\lim _{\ell \rightarrow \infty} \sum_{i=1}^{n_{\ell}} I_{i}\left(B_{\ell}\right)^{2}=0,
$$

then $\left(B_{\ell}\right)_{\ell \in \mathbb{N}}$ is asymptotically noise sensitive.

In fact, BKS only proved this theorem for the uniform measure (i.e., in the case $p=1 / 2$ ). The general result, including the case of biased measure (assuming the bias $p$ does not depend on $n$ ), was proved recently by Keller and Kindler [K-K], who moreover established a quantitative version of this result. The proof of this quantitative bound uses the Fourier-Walsh expansion of functions of the discrete cube together with hypercontractive bounds to control the tails of chaos in the expansion (hypercontractivity is actually also at the root of the asymptotic Theorem 1.11). The result is stated below as (2), but before we present the analogous continuous version which has a similar form.

The quantitative version of $[\mathrm{K}-\mathrm{K}]$ has been extended to the continuous Gaussian setting by Keller, Mossel and Sen [K-M-S2]. To state the result, we need to introduce the corresponding definitions. Let $d \mu(x)=e^{-|x|^{2} / 2} \frac{d x}{(2 \pi)^{n / 2}}$ be the canonical Gaussian measure on $\mathbb{R}^{n}$. For $W, W^{\prime}$ independent with distribution $\mu$, and $\eta>0$, set

$$
W^{\eta}=\sqrt{1-\eta^{2}} W+\eta W^{\prime}
$$


so that $\left(W, W^{\eta}\right)$ is a $\sqrt{1-\eta^{2}}$-correlated Gaussian vector. For $f: \mathbb{R}^{n} \rightarrow \mathbb{R}$, providing that $\|f(W)\|_{L^{2}(\mu)}<\infty$, define the Gaussian noise stability of $f$ as

$$
\mathcal{S}_{\eta}^{\mathcal{G}}(f)=\mathbb{E}_{\mu}\left[f(W) f\left(W^{\eta}\right)\right]-\mathbb{E}_{\mu}[f(W)]^{2} .
$$

Similarly, for a (Borel measurable) subset $A \subset \mathbb{R}^{n}$, set

$$
\mathcal{S}_{\eta}^{\mathcal{G}}(A)=\mathcal{S}_{\eta}^{\mathcal{G}}\left(\mathbf{1}_{A}\right)
$$

In order to discuss the analogue of Theorem 1.1 in the Gaussian case, it is necessary to define the influence of the $i$-th coordinate on a subset $A \subset \mathbb{R}^{n}$ in this context. To this aim, in a continuous setting, the authors of [K-M-S2] introduce the notion of geometric influence defined by

$$
I_{i}^{\mathcal{G}}(A)=\mathbb{E}_{x}\left[\mu^{+}\left(A_{i}^{x}\right)\right] .
$$

In the latter expression, $A_{i}^{x} \subset \mathbb{R}$ is the restriction of $A$ along the fibre of $x=\left(x_{1}, \ldots, x_{n}\right) \in \mathbb{R}^{n}$, that is

$$
A_{i}^{x}=\left\{y \in \mathbb{R},\left(x_{1}, \ldots, x_{i-1}, y, x_{i+1}, \ldots, x_{n}\right) \in A\right\}
$$

and $\mu^{+}$denotes the lower Minkowski content, that is for any Borel measurable set $D \subset \mathbb{R}$,

$$
\mu^{+}(D)=\liminf _{r \rightarrow 0} \frac{\mu(D+[-r, r])-\mu(D)}{r}
$$

(where $\mu$ here is the standard Gaussian distribution on $\mathbb{R}$ ).

From a more intuitive point of view, for each $i \in\{1, \ldots, n\}, I_{i}^{\mathcal{G}}(A)$ is obtained as a limit of $\left\|\partial_{i} f_{\varepsilon_{k}}\right\|_{L^{1}(\mu)}$ for a sequence of smooth functions $\left(f_{\varepsilon_{k}}\right)_{\varepsilon_{k} \geq 0}$ such that $\lim _{\varepsilon_{k} \rightarrow 0} f_{\varepsilon_{k}}=\mathbf{1}_{A}$.

We refer the reader to [K-M-S1], K-M-S2] for further developments on geometric influences and their applications.

In both the cube (with bias $p$ ) and Gaussian settings, the quantitative noise sensitivity bounds of [K-K] and [K-M-S2] may be then expressed in the following form: for any sets $A \subset\{-1,1\}^{n}$, and any $\eta \in(0,1)$,

$$
\mathcal{S}_{\eta}^{c}(A) \leq C_{1}\left(\sum_{i=1}^{n} I_{i}(A)^{2}\right)^{C_{2}(p) \eta}
$$

for the discrete cube where $C_{1}$ is numerical, and for any sets $A \subset \mathbb{R}^{n}$ and any $\eta \in(0,1)$,

$$
\mathcal{S}_{\eta}^{\mathcal{G}}(A) \leq C_{1}\left(\sum_{i=1}^{n}\left(I_{i}^{\mathcal{G}}(A)\right)^{2}\right)^{C_{2} \eta^{2}}
$$

for the Gaussian space, where $C_{1}, C_{2}$ are some positive numerical constants. These results actually are proved in theirs functional forms, that is for any bounded (by 1 ) function $f$ defined respectively in the discrete cube and in the Gaussian space ( $C^{1}$ smooth in the latter case), it holds

$$
\mathcal{S}_{\eta}^{c}(f) \leq C_{1}\left(\sum_{i=1}^{n} I_{i}(f)^{2}\right)^{C_{2} \eta} \quad \text { and } \quad \mathcal{S}_{\eta}^{\mathcal{G}}(f) \leq C_{1}\left(\sum_{i=1}^{n}\left\|\partial_{i} f\right\|_{1}^{2}\right)^{C_{2} \eta^{2}}
$$

The results in $\mathrm{K}-\mathrm{K}$ ] and $\mathrm{K}-\mathrm{M}-\mathrm{S} 2$ actually extend for functions in $L^{2}(\mu)$. Indeed, the proof of the Gaussian result in [K-M-S2 relies on the result of the discrete cube in $\mathrm{K}-\mathrm{K}$ together with an 
appropriate Central Limit Theorem argument. A close inspection of the arguments from [K-K] actually reveals that the Fourier-Walsh decomposition approach may be adapted to a Fourier-Hermite decomposition in the Gaussian case so to yield the same conclusion. Therefore, the boundedness of the functions can be weakened to obtain the same result for functions in $L^{2}(\mu)$.

The Fourier decomposition approach is however somewhat limited to the examples of the discrete cube and the Gaussian space. Indeed, a key property is the fact that the elements of the orthogonal basis of the underlying space are eigenvectors of the corresponding semigroup. Spaces satisfying such a property have been characterized in Ma. In this paper, we develop a new, simpler, proof of these quantitative relationships (2) and (3) which moreover applies to more general examples. Once again, the main ingredient will be hypercontractivity as in [B-K-S, $\mathrm{K}-\mathrm{K}$, [K-M-S2]. The starting point of the proof follows closely the work of CE-L which generalizes Talagrand's inequality of [T1 for more general models. The framework of [CE-L applies in a rather general context where hypercontractivity holds together with specific commutation properties. It covers the product of (strictly) log-concave probability measures and also discrete examples, including the biased discrete cube or, more general Schreier or Cayley graphs.

In this framework, we will establish quantitative relationships between noise stability and influences strengthening therefore the results of [B-K-S], $[\mathrm{K}-\mathrm{K}]$ and $[\mathrm{K}-\mathrm{M}-\mathrm{S} 2$, under furthermore weaker assumptions. The proposed simpler and more efficient proof relies on semigroup decompositions and cut-off arguments.

The general setting contains two main illustrations, probability measures on finite state spaces that are invariant for some Markov kernel and continuous product probability measures on $\mathbb{R}^{n}$ where each measure is of the form $d \mu(x)=e^{-V(x)} d x$ for $V$ a smooth potential. The following is a sample illustration of the results of this work (in the continuous setting). More complete statements will be presented in the process of the paper.

Theorem 1.2. Let $\left(\mathbb{R}^{n}, \mu^{\otimes n}\right)$ with $\mu$ a probability measure on $\mathbb{R}$ of the form $d \mu(x)=e^{-v(x)} d x$ where $v^{\prime \prime} \geq c$ uniformly for some $c>0$. Denote by $\mathcal{S}_{\eta}$ the noise stability with respect to $\eta$ in this context. Then, for any $\eta \in[0,1)$, and any Borel measurable set $A \in \mathbb{R}^{n}$, there exists positive constants $C, c_{1}$ depending only on $\mathrm{c}$ such that

$$
\mathcal{S}_{\eta}(A) \leq C\left(\sum_{i=1}^{n}\left(I_{i}^{\mathcal{G}}(A)\right)^{2}\right)^{c_{1} \eta^{2}} \mu^{\otimes n}(A)^{2-2 c_{1} \eta^{2}} .
$$

The generalized definition of the noise stability in this context will be given in the next section. In particular, for the standard Gaussian measure, $\mathcal{S}_{\eta}=\mathcal{S}_{\eta}^{\mathcal{G}}$ and so we recover the quantitative estimate (3) established by Keller, Mossel and Sen.

With a common scheme of proof, an analogous statement is established for discrete models covering in particular the discrete cube endowed with any biased measure $\left(p \delta_{-1}+(1-p) \delta_{1}\right)^{\otimes n}$. The precise setting and the according notion of influence will be presented in subsection 2.3.

Theorem 1.3. Let $(\Omega, \mu)=\left(\Omega_{1} \times \ldots \times \Omega_{n}, \mu_{1} \otimes \ldots \otimes \mu_{n}\right)$ be a Cartesian product of finite probability spaces. Denote by $\mathcal{S}_{\eta}$ the noise stability with respect to $\eta$ in this context. Then, for any $\eta \in[0,1)$, and any set $A \subset \Omega$, there exists some numerical constants $C, c_{1}>0$ such that

$$
\mathcal{S}_{\eta}(A) \leq C\left(\sum_{i=1}^{n}\left(I_{i}(A)\right)^{2}\right)^{c_{1} \frac{\rho}{\lambda} \eta} \mu(A)^{2-2 \frac{\rho}{\lambda} c_{1} \eta},
$$

where $\lambda$ and $\rho$ designed respectively the spectral gap and the Sobolev logarithmic constants of the product graph $\Omega$. 
The definitions of $\lambda$ and $\rho$ will be given in subsections 2.4 and 2.5. In particular if $\Omega=\{-1,1\}^{n}$, $\mu=\nu, \mathcal{S}_{\eta}=\mathcal{S}_{\eta}^{c}$ and we will see that $\rho=2 \frac{\log p-\log (1-p)}{2 p-1}, \lambda=1$ so that the above theorem extends the quantitative estimate (2) of Keller and Kindler.

The note is organized as follows. In Section 2, we first describe a convenient abstract framework and recall some basic facts about Markov semigroups that will be used in the proofs of our results. Then, in subsection 2.1,2.2 and 2.3, after developing the examples of the Gaussian space and the discrete cube, we present the general setting in which our results fall in. Then, we present our generalized definition of noise stability in subsection 2.4 and finally in subsection 2.5 we describe further tools required in the proofs of our results, in particular the hypercontractive property. In Section 3, we establish the announced result on $\left(\mathbb{R}^{n}, d \mu(x)=\bigotimes_{i=1}^{m} e^{-V_{i}(x)} d x\right)$ when the potentials $V_{i}$ are convex. This more general result encircles Theorem 1.2. Section 4 is devoted to the discrete case. Firstly, we focus on product spaces, proving Theorem 1.3 in a slightly more general form, and then we turn to the case of Cayley or Schreier graphs. In particular, we prove that the analogous result holds for the slices of the Boolean cube. We then briefly conclude in Section 5 with a similar inequality on the Euclidean spheres.

\section{A general framework}

This section presents the framework and the main tools that will be required in the proofs. The setting emphasized here is pretty general, but for the sake of clarity, we discuss in subsections the two main cases that are at the starting point of this investigation, i.e. the Gaussian space and the discrete cube endowed with uniform measure.

Let $(\Omega, \mathcal{A}, \mu)$ be a probability space. For a function $f: \Omega \rightarrow \mathbb{R}$ in $L^{2}(\mu)$, denote its variance with respect to $\mu$ by

$$
\operatorname{Var}_{\mu}(f)=\int_{\Omega} f^{2} d \mu-\left(\int_{\Omega} f d \mu\right)^{2} .
$$

In the same way, if $f \geq 0$, provided it is well defined, we denote its entropy with respect to $\mu$ by

$$
\operatorname{Ent}_{\mu}(f)=\int_{\Omega} f \log f d \mu-\int_{\Omega} f d \mu \log \left(\int_{\Omega} f d \mu\right) .
$$

The main argument of the proof will be based on interpolation along a Markov semigroup with invariant measure $\mu$. We refer to the general references [Ba], Aal], B-G-L] for complete accounts on Markov semigroups. For the reader's convenience, we briefly recall here a few basic aspects, illustrated next on the two basic model examples.

A family $\left(P_{t}\right)_{t \geq 0}$ of operators acting on a domain $\mathcal{D}$ of functions on $\Omega$ is said to be a semigroup if $P_{0}=\mathrm{Id}$ and, for all $s, t \geq 0, P_{t+s}=P_{t} \circ P_{s}$. The semigroup $\left(P_{t}\right)_{t \geq 0}$ is said to be Markov if for all $t \geq 0, P_{t} \mathbf{1}=\mathbf{1}$. The infinitesimal generator $L$ of $\left(P_{t}\right)_{t \geq 0}$ is defined by

$$
\forall f \in \mathcal{D}_{2}(L), L f:=\lim _{t \rightarrow 0} \frac{P_{t} f-f}{t}
$$

where the Dirichlet domain $\mathcal{D}_{2}(L) \subset \mathcal{D}$ is the set of all functions $f$ in $L^{2}(\mu)$ for which the above limit exists. Conversely, $L$ and $\mathcal{D}_{2}(L)$ completely determine $\left(P_{t}\right)_{t \geq 0}$. By definition and the semigroup property $\frac{\partial}{\partial t} P_{t} f=L P_{t} f$ and $P_{0} f=f$, justifying the intuitive notation $P_{t}=e^{t L}$.

Given such a Markov semigroup $\left(P_{t}\right)_{t \geq 0}$, the measure $\mu$ is said to be reversible with respect to $\left(P_{t}\right)_{t \geq 0}$ if

$$
\forall f, g \in L^{2}(\mu), \int_{\Omega} f L g d \mu=\int_{\Omega} g L f d \mu
$$


and invariant with respect to $\left(P_{t}\right)_{t \geq 0}$ if

$$
\forall f \in L^{1}(\mu), \int_{\Omega} P_{t} f d \mu=\int_{\Omega} f d \mu .
$$

The Dirichlet form associated to $(L, \mu)$ is the bilinear symmetric operator

$$
\mathcal{E}(f, g)=\int_{\Omega} f(-L g) d \mu
$$

on suitable real-valued functions $f, g$ in the Dirichlet domain.

Finally, it will be assumed moreover that $\left(P_{t}\right)_{t \geq 0}$ is ergodic with respect to $\mu$, which means that $\mu$ a-e, $P_{t} f \rightarrow \int_{\Omega} f d \mu$ as $t \rightarrow \infty$. We notice then - as a basic starting point of the future investigation - that the variance of function $f$ with respect to $\mu$ can be represented via the semigroup as

$$
\operatorname{Var}_{\mu}(f)=\int_{\Omega} f^{2} d \mu-\left(\int_{\Omega} f d \mu\right)^{2}=\lim _{t \rightarrow \infty}\left(\int_{\Omega}\left(P_{0} f\right)^{2} d \mu-\int_{\Omega}\left(P_{t} f\right)^{2} d \mu\right) .
$$

The following paragraphs aim at illustrating this general set-up by examples of interest. The first one discusses the Gaussian model, and its extension to log-concave measures. The next ones deal with the discrete cube and more general discrete models attached to Markov chains.

\subsection{The Gaussian space and continuous setting}

Let $d \mu(x)=e^{-|x|^{2} / 2} \frac{d x}{(2 \pi)^{n / 2}}$ be the standard Gaussian measure on $\Omega=\mathbb{R}^{n}$, and consider the OrnsteinUhlenbeck semigroup acting on suitable functions $f: \mathbb{R}^{n} \rightarrow \mathbb{R}$ as

$$
P_{t} f(x)=\int_{\mathbb{R}^{n}} f\left(e^{-t} x+\sqrt{1-e^{-2 t}} y\right) d \mu(y), \quad t \geq 0, x \in \mathbb{R}^{n} .
$$

As it is classical, (cf. e.g. [Ba], p. 4), the generator of the Ornstein-Uhlenbeck semigroup $\left(P_{t}\right)_{t \geq 0}$ is given by $L=\Delta-x \cdot \nabla$. The Ornstein-Uhlenbeck semigroup $\left(P_{t}\right)_{t \geq 0}$ is invariant and symmetric with respect to $\mu$, and ergodic (as easily checked on the previous integral representation). That is, for any functions $f, g \in L^{2}(\mu)$ and any $t \geq 0, \int_{\mathbb{R}^{n}} f P_{t} g d \mu=\int_{\mathbb{R}^{n}} P_{t} f g d \mu$, and $P_{t} f \rightarrow f\left(\right.$ in $\left.L^{2}(\mu)\right)$ as $t \rightarrow \infty$. The associated Dirichlet domain of contains $L^{2}(\mu) \cap C^{2}\left(\mathbb{R}^{n}\right)$, and it follows from integration by parts that for $C^{2}$ functions $f, g$ on $\mathbb{R}^{n}$,

$$
\mathcal{E}(f, g)=\int_{\mathbb{R}^{n}} f(-L g) d \mu=\int_{\mathbb{R}^{n}} \nabla f \cdot \nabla g d \mu .
$$

In particular, we have the following decomposition of the Dirichlet form along directions as

$$
\mathcal{E}(f, f)=\int_{\mathbb{R}^{n}}|\nabla f|^{2} d \mu=\sum_{i=1}^{n} \int_{\mathbb{R}^{n}}\left(\partial_{i} f\right)^{2} d \mu .
$$

According to these properties, it is immediately checked that the (Gaussian) noise stability $\mathcal{S}_{\eta}^{\mathcal{G}}(f)$ of a function $f: \mathbb{R}^{n} \rightarrow \mathbb{R}$ as described in the introduction may be reinterpreted in terms of the semigroup in the following way

Lemma 2.1. For $f: \mathbb{R}^{n} \rightarrow \mathbb{R}$ and $\eta>0$,

$$
\mathcal{S}_{\eta}^{\mathcal{G}}(f)=\operatorname{Var}_{\mu}\left(P_{t / 2} f\right)
$$

with $e^{-t}=\sqrt{1-\eta^{2}}$. 
The preceding Gaussian example may be amplified along the same lines to cover families of log-concave measures on $\mathbb{R}^{n}$. Indeed, let $d \mu(x)=e^{-V(x)} d x$ be a probability measure on the Borel sets of $\mathbb{R}^{n}$ where $V: \mathbb{R}^{n} \rightarrow \mathbb{R}$ is a smooth potential, invariant and symmetric with respect to the second order diffusion operator $L=\Delta-\nabla V \cdot \nabla$ with associated semigroup $P_{t}=e^{t L}, t \geq 0$. As in the Gaussian case, integration by parts yields, for smooth functions $f, g$ on $\mathbb{R}^{n}$,

$$
\mathcal{E}(f, g)=\int_{\mathbb{R}^{n}} f(-L g) d \mu=\int_{\mathbb{R}^{n}} \nabla f \cdot \nabla g d \mu
$$

and therefore a similar decomposition of the Dirichlet form $\mathcal{E}(f, f)$.

We will be concerned more generally with products of such measures, namely $\mu=\bigotimes_{i=1}^{m} \mu_{i}$ on $\mathbb{R}^{n}=\mathbb{R}^{n_{1}} \times \cdots \times \mathbb{R}^{n_{m}}$ where, for $i=1, \ldots, m, d \mu_{i}(x)$ is of the form $e^{-V_{i}(x)} d x$ with $V_{i}: \mathbb{R}^{n_{i}} \rightarrow \mathbb{R}$ some smooth potential. The product generator $L$ of the $L_{i}$ is given by

$$
L=\sum_{i=1}^{m} \operatorname{Id}_{\mathbb{R}^{n_{1}}} \otimes \cdots \otimes \operatorname{Id}_{\mathbb{R}^{n_{i-1}}} \otimes L_{i} \otimes \operatorname{Id}_{\mathbb{R}^{n_{i+1}}} \otimes \cdots \otimes \operatorname{Id}_{\mathbb{R}^{n_{m}}}
$$

with associated (product) semigroup $\left(P_{t}\right)_{t \geq 0}$. Setting $\nabla_{i}$ for the gradient in the direction $\mathbb{R}^{n_{i}}$, the Dirichlet form is decomposed into

$$
\mathcal{E}(f, f)=\int_{\mathbb{R}^{n}}|\nabla f|^{2} d \mu=\sum_{i=1}^{m} \int_{\mathbb{R}^{n}}\left|\nabla_{i} f\right|^{2} d \mu .
$$

In this context, we may then state the classical decomposition of the variance (and accordingly of noise stability) along the semigroup which will be the starting point of our investigation.

Lemma 2.2. For every smooth $f: \mathbb{R}^{n} \rightarrow \mathbb{R}$ and every $t \geq 0$,

$$
\operatorname{Var}_{\mu}\left(P_{t} f\right)=2 \int_{t}^{\infty} \sum_{i=1}^{m} \int_{\mathbb{R}^{n}}\left|\nabla_{i} P_{s} f\right|^{2} d \mu d s
$$

Proof. By ergodicity and the fundamental theorem of calculus,

$$
\begin{aligned}
\operatorname{Var}_{\mu}\left(P_{t} f\right) & =\int_{\mathbb{R}^{n}}\left(P_{t} f\right)^{2} d \mu-\left(\int_{\mathbb{R}^{n}} f d \mu\right)^{2} \\
& =-\int_{t}^{\infty} \frac{d}{d s} \int_{\mathbb{R}^{n}}\left(P_{s} f\right)^{2} d \mu d s \\
& =-2 \int_{t}^{\infty} \int_{\mathbb{R}^{n}} P_{s} f L\left(P_{s} f\right) d \mu d s
\end{aligned}
$$

Then, after integration by parts,

$$
\operatorname{Var}_{\mu}\left(P_{t} f\right)=2 \int_{t}^{\infty} \mathcal{E}\left(P_{s} f, P_{s} f\right) d s=2 \int_{t}^{\infty} \sum_{i=1}^{m} \int_{\mathbb{R}^{n}}\left|\nabla_{i} P_{s} f\right|^{2} d \mu d s
$$

from which the lemma follows. 


\section{$2.2 \quad$ The discrete cube}

On the discrete cube $C_{n}=\{-1,1\}^{n}$, let us consider first the case of the uniform measure $\nu\left(p=\frac{1}{2}\right)$. The biased case is an adaptation of this particular case, as developed below. Consider the BonamiBeckner semigroup $\left(T_{t}\right)_{t \geq 0}$ acting on the discrete cube given in its explicit form by

$$
T_{t} f(x)=\int_{C_{n}} f(\omega) \prod_{i=1}^{n}\left(1+e^{-t} x_{i} \omega_{i}\right) d \nu(\omega), \quad t \geq 0, x \in C_{n} .
$$

It is classical and easily checked that $\left(T_{t}\right)_{t \geq 0}$ is a Markov semigroup with invariant and reversible probability measure $\nu$. Its generator $L$ may be described as

$$
L=\frac{1}{2} \sum_{i=1}^{n} D_{i}
$$

where $D_{i}$ the $i$-th derivative of $f: C_{n} \rightarrow \mathbb{R}$ defined by $D_{i}(f)(x)=f\left(\tau_{i} x\right)-f(x)$ with $x=\left(x_{1}, \ldots, x_{n}\right)$ and $\tau_{i} x$ as in the introduction.

It is actually of some interest to emphasize the latter properties in the Fourier-Walsh decomposition. Denote $[n]$ for $\{1, \ldots, n\} . L^{2}\left(C_{n}, \nu\right)$ is an Euclidean space with respect to the standard scalar product $\langle\cdot, \cdot\rangle_{L^{2}(\nu)}$. It is well known (see e.g. [T1]) that there is an orthogonal basis, the Fourier-Walsh basis $\left(W_{S}\right)_{S \subset[n]}$ defined by $W_{S}(x)=\prod_{i \in S} x_{i}$. Each function $f \in L^{2}(\nu)$ can then be decomposed into $f=\sum_{S \subset[n]} \hat{f}(S) W_{s}$ with $\hat{f}(S)=\left\langle f, W_{S}\right\rangle_{L^{2}(\nu)}$. Using that

$$
\prod_{i=1}^{n}\left(1+e^{-t} x_{i} \omega_{i}\right)=\sum_{S \subset[n]} e^{-t|S|} \prod_{i \in S} x_{i} \omega_{i}=\sum_{S \subset[n]} e^{-t|S|} W_{S}(x) W_{S}(\omega)
$$

with $|S|$ to be the cardinal of the set $S$, it implies that

$$
T_{t}(f)=\sum_{S \subset[n]} e^{-t|S|} \hat{f}(S) W_{s}
$$

for every $t>0$. The semigroup property then easily follows. Moreover,

$$
L W_{S}(x)=-\frac{1}{2} \sum_{i=1}^{n}\left(W_{S}(x)-W_{S}\left(\tau_{i} x\right)\right)=-|S| W_{S}(x)
$$

since $W_{S}(x)-W_{S}\left(\tau_{i} x\right)=2 W_{S}(x) 1_{i \in S}(x)$ from which $L$ is seen as the generator of $\left(T_{t}\right)_{t \geq 0}$.

The associated Dirichlet domain is $L^{2}(\nu)$ since there are no regularity assumptions. Notice that the Dirichlet form $\mathcal{E}$ may again be decomposed along directions by

$$
\mathcal{E}(f, f)=\frac{1}{4} \sum_{i=1}^{n} \int_{C^{n}}\left|D_{i}(f)\right|^{2} d \nu .
$$

Besides, for a real-valued function $f$ and $i=1, \ldots, n$, the influence $I_{i}(f)$ of the $i$-th coordinate on the function $f$ as presented in the introduction is represented as

$$
I_{i}(f)=\left\|D_{i} f\right\|_{L^{1}(\nu)} \cdot
$$

With this background, as in the Gaussian space, the noise stability may be expressed in terms of the semigroup $\left(T_{t}\right)_{t \geq 0}$. 
Lemma 2.3. For $f: C_{n} \rightarrow \mathbb{R}$ and $\eta>0$,

$$
S_{\eta}^{c}(f)=\operatorname{Var}_{\nu}\left(P_{t / 2} f\right),
$$

with $e^{-t}=1-\eta$.

The preceding construction may be developed similarly on the biased case, i.e. when the cube is endowed with $\nu_{p}=\left(p \delta_{-1}+q \delta_{1}\right)^{\otimes n}$, where $p+q=1$. The Fourier-Walsh basis $\left(\omega_{S}\right)_{S \subset[n]}$ is given here by

$$
\left(\left(\frac{p}{q}\right)^{(n-|S|) / 2} \prod_{i \in S} x_{i}\right)_{S \subset[n]} .
$$

Set $L=2 p q \sum_{i=1}^{n} D_{i}$. Arguing as in the case $p=\frac{1}{2}$, if we set $T_{t}^{p} f=\sum_{S \subset[n]} e^{-t|S|} \hat{f}(S) \omega_{S}$, then $L$ is the generator of $\left(T_{t}^{p}\right)_{t \geq 0}$. In the same way

$$
\mathcal{S}_{\eta}(f)=\operatorname{Var}_{\nu_{p}}\left(T_{t / 2}^{p} f\right)
$$

In addition, the Dirichlet form $\mathcal{E}$ takes the same form

$$
\mathcal{E}(f, f)=p q \sum_{i=1}^{n} \int_{\{-1,1\}^{n}}\left|D_{i}(f)\right|^{2} d \nu_{p} .
$$

For both the uniform and biased cube, the decomposition of the variance along the semigroup is similar to the one emphasized in the continuous setting in Lemma 2.2.

Lemma 2.4. For every $f: C_{n} \rightarrow \mathbb{R}$, every $t \geq 0$, and every $p \in(0,1)$,

$$
\operatorname{Var}_{\nu_{p}}\left(P_{t} f\right)=2 \int_{t}^{\infty} \sum_{i=1}^{n} \int_{C^{n}}\left|D_{i} T_{s}^{p} f\right|^{2} d \nu d s
$$

\subsection{General discrete case}

In this subsection, we discuss extensions of the discrete cube model to general discrete spaces, that we assume to be finite (as it will be the case for concrete illustrations).

Let $\Omega$ be a finite space with probability measure $\mu$ on which there is a Markov kernel $K$, invariant and reversible with respect to $\mu$, i.e. such that

$$
\forall(x, y) \in \Omega^{2}, \quad \sum_{x \in \Omega} K(x, y) \mu(x)=\mu(y) \quad \text { and } \quad K(x, y) \mu(x)=K(y, x) \mu(y) .
$$

Define $L$ by $L=K-I d$, generator of the semigroup $P_{t}=e^{t L}, t \geq 0$. The associated Dirichlet form is given by

$$
\mathcal{E}(f, g)=\int_{\Omega} f(-L g) d \mu=\frac{1}{2} \sum_{x, y \in \Omega}(f(x)-f(y))(g(x)-g(y)) K(x, y) \mu(y)
$$

for functions $f, g$ on $\Omega$. 
The discrete cube model enters this setting by a suitable choice of the kernel $K$. Consider namely the operator given by $L f=\int_{\Omega} f d \mu-f$, i.e $K f=\int_{\Omega} f d \mu$, or $K=\operatorname{diag}(\mu(x))_{x \in \Omega}$. In particular, a simple computation shows that

$$
\operatorname{Var}_{\mu}(f)=\int_{\Omega} f(-L f) d \mu=\mathcal{E}(f, f) .
$$

An interesting instance of the preceding, extending the case of the Boolean cube, is given by such product spaces with product measures

$$
\Omega=\Omega_{1} \times \cdots \times \Omega_{n} \quad \text { with } \quad \mu=\mu_{1} \otimes \cdots \otimes \mu_{n},
$$

when we take product of the above Markov operators. That is, set, for $i=1, \ldots, n$, and $f: \Omega \rightarrow \mathbb{R}$ $L_{i} f=\int_{\Omega_{i}} f d \mu_{i}-f$ and consider the generator on the product space given by

$$
L f=\sum_{i=1}^{n} L_{i} f .
$$

In this case the Dirichlet form $\mathcal{E}$ may be decomposed as

$$
\mathcal{E}(f, f)=\sum_{i=1}^{n} \int_{\Omega_{i}} L_{i}(f)^{2} d \mu_{i}
$$

This setting, which will be referred to in the following as discrete product structure, covers the cube with $\Omega_{1}=\cdots=\Omega_{n}=\{-1,1\}$, each of which being equipped with the measure $p \delta_{-1}+q \delta_{1}$. Among other relevant examples one can take for any $i, \Omega_{i}=\mathbb{Z} / q \mathbb{Z}$ for any $q \geq 3$ endowed with uniform measure. In this context, the influence of the $i$-th coordinate is naturally defined as $\left\|L_{i} f\right\|_{1}$ (notice that both definitions over the Boolean cube agree up to a constant depending on the bias $p$ ).

The preceding can be extended to more general Cayley or Schreier graphs (see [CE-L, O-W]), covering therefore non-product examples. Let $G$ be a (finite) group for which there is a finite set of generators $S$, symmetric, i.e. $S^{-1}=S$, and stable by conjugacy. Assume that $G$ is acting transitively on a finite set $\Omega$ and denote for $g \in G, x \in \Omega, x^{g}$ the action of $g$ on $x$. The associated Schreier graph is the graph of vertices $\Omega$ and edges $(x, y)$ if and only if there is a $s \in S$ such that $y=x^{s}$. A Cayley graph corresponds to the particular case $G=\Omega$, consisting therefore of the set of vertices $(g)_{g \in G}$ with edges $(g, g s)_{g \in G, s \in S}$. One basic example is the symmetric group $\mathfrak{S}_{n}$ with generating set transpositions $\mathcal{T}_{n}$.

Given a Cayley of Schreier graph $G$, consider the transition kernel $K$ given by

$$
K\left(g_{1}, g_{2}\right)=\frac{1}{|S|} 1_{S}\left(g_{1} g_{2}^{-1}\right), \quad g_{1}, g_{2} \in G,
$$

corresponding to the random walk to nearest neighbour and the uniform measure $\mu$ on $G$. Again, it generates the family of semigroups $\left(P_{t}=e^{t L}\right)_{t \geq 0}$, with $L=K-I d$. The associated Dirichlet forms $\mathcal{E}$ can be written as

$$
\mathcal{E}(f, f)=\frac{1}{2|S|} \sum_{g \in G} \sum_{s \in S}[f(g s)-f(g)]^{2} \mu(g)=\frac{1}{2|S|} \sum_{s \in S}\left\|D_{s} f\right\|_{L^{2}(G)}^{2},
$$

where $D_{s} f: g \mapsto f(g s)-f(g)$ in the Cayley graph case and

$$
\mathcal{E}(f, f)=\frac{1}{2|S|} \sum_{x \in \Omega} \sum_{s \in S}\left[f\left(x^{s}\right)-f(x)\right]^{2} \mu(x)=\frac{1}{2|S|} \sum_{s \in S}\left\|D_{s} f\right\|_{L^{2}(\Omega)}^{2},
$$


where $D_{s} f: x \mapsto f\left(x^{s}\right)-f(x)$ in the Schreier graph case.

Note finally that the influence of a generator element $s \in S$ on a function $f$ is then naturally defined in this context by $\left\|D_{s} f\right\|_{1}$.

\subsection{The generalized version of noise stability}

In this section, we extend the definition of noise stability to the different models presented in the preceding subsections.

Consider therefore the preceding setting of a probability space $(\Omega, \mathcal{A}, \mu)$ equipped with a semigroup $\left(P_{t}\right)_{t \geq 0}$ with generator $L$ and Dirichlet form $\mathcal{E}$, invariant and symmetric with respect to $\mu$.

Say that the couple $(L, \mu)$ satisfies a spectral gap, or Poincaré, inequality whenever there exists $\lambda>0$ such that

$$
\lambda \operatorname{Var}_{\mu}(f) \leq \mathcal{E}(f, f)
$$

for every function $f$ on the Dirichlet domain. The spectral gap constant is the largest $\lambda$ such that (8) holds. It is equivalent to the fact that for every functions $f$ in $L^{2}(\mu)$, and every $t>0$,

$$
\operatorname{Var}_{\mu}\left(P_{t} f\right) \leq e^{-2 \lambda t} \operatorname{Var}_{\mu}(f) \text {. }
$$

This follows immediately from Gronwall's lemma since $\frac{d}{d t} \operatorname{Var}_{\mu}\left(P_{t} f\right)=\frac{d}{d t}\left\|P_{t} f\right\|_{2}^{2}=-2 \mathcal{E}\left(P_{t} f, P_{t} f\right)$. For further purposes, it is then not hard to check (see [CE-L]), that the spectral gap inequality with constant $\lambda$ is equivalent to the fact that for every centered function $f$ (i.e. $\int_{\Omega} f d \mu=0$ ),

$$
\forall t>0, \operatorname{Var}_{\mu}(f) \leq \frac{1}{1-e^{-\lambda t}}\left(\|f\|_{2}^{2}-\left\|P_{t} f\right\|_{2}^{2}\right)
$$

As standard examples, $\lambda=1$ for the standard Gaussian measure on $\mathbb{R}^{n}$ and similarly for the discrete cube equipped with any biased measure $\nu$. As a result, from Lemma 2.1 and Lemma 2.3. the spectral gap inequality in its formulation (9) implies

$$
\mathcal{S}_{\eta}^{c}(f) \leq(1-\eta)\|f\|_{2}^{2} \quad\left(\operatorname{resp} . \mathcal{S}_{\eta}^{\mathcal{G}}(f) \leq \sqrt{1-\eta^{2}}\|f\|_{2}^{2}\right)
$$

for (centered) functions of the discrete cube (resp. of the Gaussian space). Thus, for a sequence of (centered) functions $\left(f_{n}\right)_{n \geq 0}$ defined on the discrete cube or on the Gaussian space such that $\inf _{n \geq 0}\left\|f_{n}\right\|_{2}>0$, the spectral gap inequality does not predict if it is a noise sensitive sequence.

We then extend the definition of noise stability of sets/functions in our both discrete and continuous setting in order to preserve this property.

Turning to log-concave measures, we give the following definition.

Definition 2.5. Let $\left(\mathbb{R}^{n}, \mu\right)$, where $\mu$ is a product a log-concave probability measures, with $\lambda$ its spectral gap constant. For a measurable function $f$ in $L^{2}(\mu)$, we define its noise stability with parameter $\eta \in[0,1)$ by

$$
\mathcal{S}_{\eta}(f)=\operatorname{Var}_{\mu}\left(P_{t} f\right)
$$

where $e^{-2 \lambda t}=\sqrt{1-\eta^{2}}$, and similarly for Borel sets $A \subset \mathbb{R}^{n}, \mathcal{S}_{\eta}(A)=\mathcal{S}_{\eta}\left(\mathbf{1}_{A}\right)$.

In the context of Schreier graphs, extending the case of the Boolean cube, the definition of noise stability is the following. 
Definition 2.6. Let $\Omega$ be a finite Schreier graph with spectral gap constant $\lambda$. For a function $f: \Omega \rightarrow \mathbb{R}$, we define its noise stability with parameter $\eta \in[0,1)$ by

$$
\mathcal{S}_{\eta}(f)=\operatorname{Var}_{\mu}\left(P_{t} f\right)
$$

where $e^{-2 \lambda t}=1-\eta$, and similarly for sets $A \subset \Omega, \mathcal{S}_{\eta}(A)=\mathcal{S}_{\eta}\left(\mathbf{1}_{A}\right)$.

Notice that, in more probabilistic terms, since $\left(P_{t}\right)_{t \geq 0}=\left(e^{-t} e^{t K}\right)_{t \geq 0}$ and by symmetry of $\left(P_{t}\right)_{t \geq 0}$ with respect to $\mu$,

$$
\mathcal{S}_{\eta}(f)=\mathbb{E}_{\mu \otimes \mu}(f(x) f(y))-\left(\mathbb{E}_{\mu} f\right)^{2},
$$

where $y$ is obtained from $x$ by acting randomly $m$ elements of the generating set $S$ where $m \sim \mathcal{P}(2 t)$ the Poisson law of parameter $t=t(\eta)$.

From subsection 2.2, the last definition agrees with $\mathcal{S}_{\eta}^{c}$ in the case of the Boolean cube. Besides, in the general case the spectral gap inequality leads by (9) to the same upper bound $\mathcal{S}_{\eta}(f) \leq$ $(1-\eta) \operatorname{Var}_{\mu}(f)$. Thus, in this general case, a quantitative relationship of the form (2) is a similar improvement upon the spectral gap inequality.

\subsection{The hypercontractive tool and the decomposition along "directions"}

In this section, we describe the main functional tool which will be used in the proof of the main results of this work. That is, the hypercontractive property of the underlying semigroups, or in its equivalent formulation logarithmic Sobolev inequalities. Besides we present in a fairly abstract setting the common decomposition of the Dirichlet form along "directions" with the associated influences with respect to these directions.

Say that $(L, \mu)$ satisfies a logarithmic Sobolev inequality whenever there exists $\rho>0$ such that

$$
\rho \operatorname{Ent}_{\mu}\left(f^{2}\right) \leq 2 \mathcal{E}(f, f)
$$

for every function $f$ on the Dirichlet domain. The logarithmic Sobolev constant is the largest $\rho>0$ such that (11) holds. Since the work of Gross [G] in the continuous setting (cf. [Ba], $\mathrm{Aal}$, [B-G-L]...) and Diaconis and Saloff-Coste [D-SC] in the discrete setting, it is known that a logarithmic Sobolev inequality is equivalent to hypercontractivity of the semigroup $\left(P_{t}\right)_{t \geq 0}$ (see $[\mathrm{N}]$ ) in the sense that for all $f \in L^{p}(\mu)$ and all $t>0,1<p<q<\infty$ with $p \geq 1+(q-1) e^{-2 \rho t}$,

$$
\left\|P_{t} f\right\|_{q} \leq\|f\|_{p}
$$

The Sobolev logarithmic constant can also be referred as the hypercontractive constant. It is classical that it is lower that the spectral gap constant, i.e. $\lambda \geq \rho$. It is also a main feature of these inequalities that they are stable by product, so that the spectral gap and logarithmic Sobolev constants of a product space are the minimum of the spectral gap and logarithmic Sobolev constants of each factor in the product. Recall that $\lambda=\rho=1$ for the standard Gaussian measure on $\mathbb{R}^{n}$ and similarly for the uniform measure on the discrete cube. For the further purposes, let us emphasize also that if $d \mu(x)=e^{-V(x)} d x$ on $\mathbb{R}^{n}$ is such that the Hessian of the potential $V$ satisfies $\operatorname{Hess}(V) \geq c>0$ (uniformly, as symmetric matrices), then $\lambda \geq \rho \geq c$. This very classical fact follows from the pioneer work of Bakry and Émery [B-E] around hypercontractive diffusions.

In each class of examples of the above subsections, a key property is the decomposition of the Dirichlet form along "directions". We will be interested into situations in which these directions commute in an appropriate sense with derivation. This may be expressed in the following abstract 
formulation, that immediately applies to the various examples of interest. Namely, assume that there is a decomposition of the Dirichlet form $\mathcal{E}$ along "directions" $i$ for some operators $\Gamma_{i}$ such that for any suitable $f$ on $\Omega$,

$$
\mathcal{E}(f, f)=\sum_{i=1}^{m} \int_{\Omega} \Gamma_{i}(f)^{2} d \mu,
$$

where the operators $\Gamma_{i}$ are commuting with the semigroup in a sense that there exists a real constant $\kappa$ such that for every $i=1, \ldots, m$

$$
\Gamma_{i}\left(P_{t} f\right) \leq e^{\kappa t} P_{t}\left(\Gamma_{i} f\right) .
$$

To illustrate this rather formal property, consider the example of the Ornstein-Uhlenbeck semigroup on $\mathbb{R}^{n}$ for which $\partial_{i} P_{t} f=e^{-t} P_{t}\left(\partial_{i} f\right)$ yielding therefore (13) with $\kappa=1$. In the framework of a logconcave measure $d \mu(x)=e^{-V(x)} d x$, it is known (see e.g [Ba], [B-G-L]) that whenever the Hessian of the potential $V$ satisfies $\operatorname{Hess}(V) \geq c$ where $c \in \mathbb{R}$, for every smooth $f: \mathbb{R}^{n} \rightarrow \mathbb{R}$ and every $t \geq 0$,

$$
\left|\nabla P_{t} f\right| \leq e^{-c t} P_{t}(|\nabla f|)
$$

Therefore, a product of log-concave measures $d \mu_{i}(x)=e^{-V_{i}(x)} d x, i=1, \ldots, m$, for which each potential $V_{i}$ satisfies $\operatorname{Hess}\left(V_{i}\right) \geq c_{i}$ with $c_{i} \in \mathbb{R}$, is another instance of the decomposition (13) with $\Gamma_{i}=\nabla_{i}$ and $\kappa=\min c_{i}$. In the discrete product setting, we may take $\Gamma_{i}=L_{i}$. On the cube, the relations $D_{i} T_{t}^{p} f=T_{t}^{p} D_{i} f$ then ensure the suitable commutation with $\kappa=0$.

Such general decompositions have been emphasized in [CE-L in connections with the study of influences. They are similarly useful here, and together with hypercontractivity will be at the root of the main conclusions. Indeed, assuming ergodicity and proceeding as in the proof of Lemma 2.2. the noise stability can then be expressed as

$$
\mathcal{S}_{\eta}(f)=\sum_{i=1}^{m} \int_{t}^{\infty} \int_{\Omega} \Gamma_{i}\left(P_{s} f\right)^{2} d \mu d s,
$$

where $t=t(\eta)$ is given as in Definition 2.5] in the continuous case or Definition 2.6] in the discrete one. Another example of interest given by the Euclidean spheres will be presented in Section 5 below.

To close this paragraph, it is worthwhile mentioning that the content of our statements are invariant under translation of the functions by a constant. Therefore, in the remaining of the paper, it will be implicitly assumed that all functions are centered.

\section{The Gaussian and log concave settings}

The section will be devoted to the proof of Theorem [1.2, actually in a more general formulation.

Consider therefore a probability measure $\mu$ on $\mathbb{R}^{n}=\mathbb{R}^{n_{1}} \times \cdots \times \mathbb{R}^{n_{m}}$ of the form $d \mu(x)=$ $\bigotimes_{i=1}^{m} e^{-V_{i}(x)} d x$ with $\operatorname{Hess}\left(V_{i}\right) \geq c>0$ for every $i=1, \ldots, m$. Recall that, if $\rho$ designs the hypercontractive constant and $\lambda$ the Poincaré constant, in this setting it holds $\lambda \geq \rho \geq c$.

Theorem 3.1. Let $\mu$ as above and let $f: \mathbb{R}^{n} \rightarrow \mathbb{R}$ be $C^{1}$-smooth and in $L^{2}(\mu)$. Then, for every $\eta \in(0,1)$,

$$
\mathcal{S}_{\eta}(f) \leq \max \left(4, \frac{4}{c}\right)\left(1-\eta^{2}\right)^{\frac{c}{4 \lambda}}\left(\sum_{i=1}^{m}\left\|\nabla_{i} f\right\|_{1}^{2}\right)^{\alpha(\eta)}\|f\|_{2}^{2-2 \alpha(\eta)}
$$


where

$$
\alpha(\eta)=\frac{1-\left(1-\eta^{2}\right)^{\frac{\rho}{4 \lambda}}}{2} .
$$

To compare with the results of [K-M-S2] in the Gaussian case (corresponding therefore to $m=n$, $n_{1}=\cdots=n_{m}=1$, and $V_{i}$ quadratic) for which $\lambda=\rho=c=1$, Theorem 3.1 indicates that for all $f: \mathbb{R}^{n} \rightarrow \mathbb{R}$ and every $\eta \in(0,1)$,

$$
\mathcal{S}_{\eta}^{\mathcal{G}}(f) \leq 4\left(1-\eta^{2}\right)^{1 / 4}\left(\sum_{i=1}^{n}\left\|\partial_{i} f\right\|_{L^{1}(\mu)}^{2}\right)^{\frac{1-\left(1-\eta^{2}\right)^{1 / 4}}{2}}\|f\|_{2}^{1+\left(1-\eta^{2}\right)^{1 / 4}} .
$$

It may always be assumed that $\sum_{i=1}^{n}\left\|\partial_{i} f\right\|_{L^{1}(\mu)}^{2} \leq\|f\|_{2}^{2}$ otherwise the above inequality is implied by the spectral gap inequality (in its formulation $\mathcal{S}_{\eta}^{\mathcal{G}}(f) \leq \sqrt{1-\eta^{2}}\|f\|_{2}^{2}$ ). As

$$
1-\left(1-\eta^{2}\right)^{1 / 4} \geq \frac{\eta^{2}}{4}
$$

it thus yields the inequality of [K-M-S2] with $C_{1}=4$ and $C_{2}=\frac{1}{8}$.

Proof of Theorem 3.1. We make the assumption that $f$ is such that

$$
\sum_{i=1}^{m}\left\|\nabla_{i} f\right\|_{1}^{2} \leq\|f\|_{2}^{2}
$$

otherwise there is nothing to prove (again, by the spectral gap inequality). We set $t=-\frac{\log \left(1-\eta^{2}\right)}{4 \lambda}$, so that $\mathcal{S}_{\eta}(f)=\operatorname{Var}_{\mu}\left(P_{t} f\right)$.

We start with Lemma 2.2 from which

$$
\operatorname{Var}_{\mu}\left(P_{t} f\right)=2 \int_{t}^{\infty} \sum_{i=1}^{m} \int_{\mathbb{R}^{n}}\left|\nabla_{i} P_{s} f\right|^{2} d \mu d s .
$$

The main step of the proof consists in the following cut-off argument. Namely, for $i=1, \ldots, m$ and $M>0$,

$$
\int_{\mathbb{R}^{n}}\left|\nabla_{i} P_{s} f\right|^{2} d \mu=\int_{\left\{\left|\nabla_{i} P_{s} f\right| \leq M\left\|\nabla_{i} f\right\|_{1}\right\}}\left|\nabla_{i} P_{s} f\right|^{2} d \mu+\int_{\left\{\left|\nabla_{i} P_{s} f\right|>M\left\|\nabla_{i} f\right\|_{1}\right\}}\left|\nabla_{i} P_{s} f\right|^{2} d \mu .
$$

The first integral is bounded from above as

$$
\begin{aligned}
\int_{\left\{\left|\nabla_{i} P_{s} f\right| \leq M\left\|\nabla_{i} f\right\|_{1}\right\}}\left|\nabla_{i} P_{s} f\right|^{2} d \mu & \leq M\left\|\nabla_{i} f\right\|_{1} \int_{\mathbb{R}^{n}}\left|\nabla_{i} P_{s} f\right| d \mu \\
& \leq M\left\|\nabla_{i} f\right\|_{1} e^{-c s} \int_{\mathbb{R}^{n}} P_{s}\left(\left|\nabla_{i} f\right|\right) d \mu
\end{aligned}
$$

where we use in the last inequality the commutation property (15) which ensures that $\left|\nabla_{i} P_{s} f\right| \leq$ $e^{-c s} P_{s}\left(\left|\nabla_{i} f\right|\right)$. Since the measure $\mu$ is invariant with respect to $\left(P_{s}\right)_{s \geq 0}$, it follows that

$$
\int_{\left\{\left|\nabla_{i} P_{s} f\right| \leq M\left\|\nabla_{i} f\right\|_{1}\right\}}\left|\nabla_{i} P_{s} f\right|^{2} d \mu \leq e^{-c s} M\left\|\nabla_{i} f\right\|_{1}^{2} .
$$


After integrating in time and summing over $i=1, \ldots, m$, we reach a first bound

$$
\sum_{i=1}^{m} \int_{t}^{\infty} \int_{\left\{\left|\nabla_{i} P_{s} f\right| \leq M\left\|\nabla_{i} f\right\|_{1}\right\}}\left|\nabla_{i} P_{s} f\right|^{2} d \mu d s \leq \frac{1}{c} e^{-c t} M \sum_{i=1}^{m}\left\|\nabla_{i} f\right\|_{1}^{2} .
$$

We now focus on bounding from above

$$
\sum_{i=1}^{m} \int_{\left\{\left|\nabla_{i} P_{s} f\right|>M\left\|\nabla_{i} f\right\|_{1}\right\}}\left|\nabla_{i} P_{s} f\right|^{2} d \mu
$$

To this task, for every $i=1, \ldots, m$, Hölder's inequality applied to $\left|\nabla_{i} P_{s} f\right|^{2}$ and $\mathbf{1}_{\left\{\left|\nabla_{i} P_{s} f\right|>M\left\|\nabla_{i} f\right\|_{1}\right\}}$ yields

$$
\int_{\left\{\left|\nabla_{i} P_{s} f\right|>M\left\|\nabla_{i} f\right\|_{1}\right\}}\left|\nabla_{i} P_{s} f\right|^{2} d \mu \leq \mu\left\{\left|\nabla_{i} P_{s} f\right|>M\left\|\nabla_{i} f\right\|_{1}\right\}^{\frac{1}{q}}\left\|\nabla_{i} P_{s} f\right\|_{2 p}^{2}
$$

for every $p, q \geq 1$ such that $\frac{1}{p}+\frac{1}{q}=1$. Yet, since $P_{s} f=P_{s / 2}\left(P_{s / 2} f\right)$, using (15) once more,

$$
\left|\nabla_{i} P_{s} f\right|^{2 p} \leq e^{-c p s}\left[P_{s / 2}\left(\left|\nabla_{i} P_{s / 2} f\right|\right)\right]^{2 p},
$$

so that by integration

$$
\left\|\nabla_{i} P_{s} f\right\|_{2 p}^{2} \leq e^{-c s}\left\|P_{s / 2}\left(\left|\nabla_{i} P_{s / 2} f\right|\right)\right\|_{2 p}^{2} .
$$

Now, the hypercontractive property (12) ensures that, if $\rho$ designs the hypercontractive constant, for $p=p(s)$ with $2 p(s)-1=e^{\rho s}$,

$$
\left\|P_{s / 2}\left(\left|\nabla_{i} P_{s / 2} f\right|\right)\right\|_{2 p}^{2} \leq\left\|\nabla_{i} P_{s / 2} f\right\|_{2}^{2} .
$$

Putting (18) and the last two inequalities together, we infer that

$$
\int_{\left\{\left|\nabla_{i} P_{s} f\right|>M\left\|\nabla_{i} f\right\|_{1}\right\}}\left|\nabla_{i} P_{s} f\right|^{2} d \mu \leq e^{-c s} \mu\left\{\left|\nabla_{i} P_{s} f\right|>M\left\|\nabla_{i} f\right\|_{1}\right\}^{\frac{1}{q(s)}}\left\|\nabla_{i} P_{s / 2} f\right\|_{2}^{2}
$$

with

$$
q(s)=\frac{p(s)}{p(s)-1}=\frac{e^{\rho s}+1}{e^{\rho s}-1} .
$$

By Markov's inequality, we further obtain, using again the commutation (15) and the invariant property of the semigroup, that

$$
\mu\left\{\left|\nabla_{i} P_{s} f\right|>M\left\|\nabla_{i} f\right\|_{1}\right\} \leq \frac{1}{M\left\|\nabla_{i} f\right\|_{1}} \int_{\mathbb{R}^{n}}\left|\nabla_{i} P_{s} f\right| d \mu \leq \frac{e^{-c s}}{M} .
$$

The above bounds (19) and (20) therefore imply

$$
\begin{aligned}
\int_{t}^{\infty} \int_{\left\{\left|\nabla_{i} P_{s} f\right|>M\left\|\nabla_{i} f\right\|_{1}\right\}}\left|\nabla_{i} P_{s} f\right|^{2} d \mu d s & \leq \int_{t}^{\infty}\left(\frac{e^{-c s}}{M}\right)^{\frac{1}{q(s)}} e^{-c s}\left\|\nabla_{i} P_{s / 2} f\right\|_{2}^{2} d s \\
& \leq e^{-c t} \int_{t}^{\infty} \frac{1}{M^{\frac{1}{q(s)}}\left\|\nabla_{i} P_{s / 2} f\right\|_{2}^{2} d s .}
\end{aligned}
$$


We then notice that the function

$$
s \mapsto \frac{1}{q(s)}=\tanh (\rho s / 2)
$$

is increasing. Hence, for every $M \geq 1$,

$$
e^{-c t} \int_{t}^{\infty} \frac{1}{M^{\frac{1}{q(s)}}}\left\|\nabla_{i} P_{s / 2} f\right\|_{2}^{2} d s \leq \frac{e^{-c t}}{M^{\frac{1}{q(t)}}} \int_{t}^{\infty}\left\|\nabla_{i} P_{s / 2} f\right\|_{2}^{2} d s .
$$

Summing over $i=1, \ldots, m$,

$$
\sum_{i=1}^{m} \int_{t}^{\infty} \int_{\left\{\left|\nabla_{i} P_{s} f\right|>M\left\|\nabla_{i} f\right\|_{1}\right\}}\left|\nabla_{i} P_{s} f\right|^{2} d \mu d s \leq \frac{e^{-c t}}{M^{\frac{1}{q(t)}}} \sum_{i=1}^{m} \int_{t}^{\infty}\left\|\nabla_{i} P_{s / 2} f\right\|_{2}^{2} d s .
$$

By Lemma 2.2 again,

$$
\sum_{i=1}^{m} \int_{t}^{\infty}\left\|\nabla_{i} P_{s / 2} f\right\|_{2}^{2} d s=\operatorname{Var}_{\mu}\left(P_{t / 2} f\right) \leq\|f\|_{2}^{2}
$$

(recall that $f$ is centered) so that

$$
\sum_{i=1}^{m} \int_{t}^{\infty} \int_{\left\{\left|\nabla_{i} P_{s} f\right|>M\left\|\nabla_{i} f\right\|_{1}\right\}}\left|\nabla_{i} P_{s} f\right|^{2} d \mu d s \leq \frac{e^{-c t}}{M^{\frac{1}{q(t)}}}\|f\|_{2}^{2} .
$$

By the decomposition (16), the two bounds (17) and (21) therefore yield

$$
\operatorname{Var}_{\mu}\left(P_{t} f\right) \leq \max \left(2, \frac{2}{c}\right) e^{-c t}\left(M \sum_{i=1}^{m}\left\|\nabla_{i} f\right\|_{1}^{2}+\frac{1}{M^{\frac{1}{q(t)}}}\|f\|_{2}^{2}\right) .
$$

Here, we recall that $M \geq 1$. Given the assumption $\|f\|_{2}^{2} \geq \sum_{i=1}^{m}\left\|\nabla_{i} f\right\|_{1}^{2}$, we can choose $M$ such that

$$
M \sum_{i=1}^{m}\left\|\nabla_{i} f\right\|_{1}^{2}=\frac{1}{M^{\frac{1}{q(t)}}}\|f\|_{2}^{2}
$$

We therefore get

$$
M^{1+\frac{1}{q(t)}}=\frac{\|f\|_{2}^{2}}{\sum_{i=1}^{m}\left\|\nabla_{i} f\right\|_{1}^{2}}
$$

so that, finally

$$
\operatorname{Var}_{\mu}\left(P_{t} f\right) \leq \max \left(4, \frac{4}{c}\right) e^{-c t}\left(\sum_{i=1}^{n}\left\|\nabla_{i} f\right\|_{1}^{2}\right)^{\frac{1}{1+q(t)}}\|f\|_{2}^{2 \frac{q(t)}{1+q(t)}} .
$$

Replacing $q(t)$ by its explicit form, and recalling $t=-\frac{\log \left(1-\eta^{2}\right)}{4 \lambda}$, we conclude to the announced claim. Theorem 3.1 is established. 


\section{The discrete setting}

\subsection{The case of Boolean cube and other discrete product spaces}

This section develops the corresponding analysis on the cube and discrete product models.

To deal with the Boolean cube $\{-1,1\}^{n}$, consider the discrete product structure as emphasized in Subsection 2.3 consisting of a product space $\Omega=\Omega_{1} \times \cdots \times=\Omega_{n}$ with product probability measure $\mu=\mu_{1} \otimes \cdots \otimes \mu_{n}$, each factor $\left(\Omega_{i}, \mu_{i}\right)$ being endowed with the Markov operator $L_{i} f=\int_{\Omega_{i}} f d \mu_{i}-f$. We recall that the Dirichlet form $\mathcal{E}$ admits the decomposition

$$
\mathcal{E}(f, f)=\sum_{i=1}^{n} \int_{\Omega_{i}} L_{i}(f)^{2} d \mu_{i}
$$

and that the equality $\operatorname{Var}_{\mu} f=\mathcal{E}(f, f)$ implies that the spectral gap constant is equal to 1 . The underlying (product) semigroup $\left(P_{t}\right)_{t \geq 0}$ will be assumed to be hypercontractive with constant $\rho$ (equivalently, each $\left(L_{i}, \mu_{i}\right)$ is hypercontractive with constant $\rho$ ).

Theorem 4.1. In the preceding setting, let $f: \Omega \rightarrow \mathbb{R}$, such that $f \in L^{2}(\mu)$. Then, if $\eta \in[0,1)$,

$$
\mathcal{S}_{\eta}(f) \leq 7\left(\sum_{i=1}^{n}\left\|L_{i} f\right\|_{1}^{2}\right)^{\alpha(\eta)}\|f\|_{2}^{2-2 \alpha(\eta)}
$$

where

$$
\alpha(\eta)=\frac{1-(1-\eta)^{\rho / 2}}{2} \geq \frac{\rho}{4} \eta
$$

Theorem 4.1 contains the result of $[\mathrm{K}-\mathrm{K}]$ for the discrete cube $\Omega=\{-1,1\}^{n}$ with $\mu=\nu_{p}=$ $\left(p \delta_{-1}+q \delta_{1}\right)^{\otimes n}$. To make the connection, notice that we can assume that

$$
\sum_{i=1}^{n}\left\|L_{i} f\right\|_{1}^{2} \leq\|f\|_{2}^{2},
$$

otherwise there is nothing to prove. With the previous notations, it is easily seen that for every $i \in\{1, \ldots, n\}$,

$$
\left\|L_{i} f\right\|_{1}=2 p q\left\|D_{i} f\right\|_{1}=2 p q I_{i}(f)
$$

Thereby, we get

$$
\mathcal{S}_{\eta}^{c}(f) \leq 7(2 p q)^{\frac{\rho}{2} \eta}\left(\sum_{i=1}^{n}\left(I_{i} f\right)^{2}\right)^{\frac{\rho}{4} \eta}\|f\|_{2}^{2-\frac{\rho}{2} \eta} .
$$

so that, since $4 p q \leq 1$,

$$
\mathcal{S}_{\eta}^{c}(f) \leq 7\left(\sum_{i=1}^{n}\left(I_{i} f\right)^{2}\right)^{\frac{\rho}{4} \eta}\|f\|_{2}^{2-\frac{\rho}{2} \eta} .
$$

On the other hand, it is known (see CE-L]) the logarithmic Sobolev constant is equal to

$$
\rho=\frac{2(p-q)}{\log p-\log q} \quad(=1 \text { if } p=q) .
$$

which amounts to the main result of $[\mathrm{K}-\mathrm{K}]$ as emphasized in the introduction. In the uniform case (i.e $p=q$ ), we have $\rho=1$ and the above result is similar to Theorem 4 of $[\mathrm{K}-\mathrm{K}$ with weaker assumptions on $f$. In the biased case, the constants are somewhat weaker for small $p$ or $q$. 
It is worth to point out that the quantitative relationship of Theorem 4.1 yields empty results when $\log p$ is of order $\log n$. In this range indeed, the theorem does not hold even qualitatively (see $[\mathrm{K}-\mathrm{K}]$.

Proof of Theorem 4.1. The scheme of proof is completely similar to the one developed in the continuous setting for Theorem 3.1. We nevertheless introduce a further trick, replacing the decomposition of the variance along the semigroup of Lemma 2.4 by its restriction to a finite domain of integration in time $[0, T]$ for some $T \geq 0$ in the form of the identity

$$
\|f\|_{2}^{2}-\left\|P_{T} f\right\|_{2}^{2}=-\int_{0}^{T} \frac{d}{d s}\left\|P_{s} f\right\|_{2}^{2} d s=2 \int_{0}^{T} \mathcal{E}\left(P_{s} f, P_{s} f\right) d s=2 \int_{0}^{T} \sum_{i=1}^{n} \int_{\Omega}\left|L_{i} P_{s} f\right|^{2} d \mu d s .
$$

Recall that if $e^{-2 t}=1-\eta, \mathcal{S}_{\eta}(f)=\operatorname{Var}_{\mu}\left(P_{t} f\right)$. Hence, by (10), for every $T>0$,

$$
\mathcal{S}_{\eta}(f) \leq \frac{2}{1-e^{-T}} \int_{t}^{t+T} \sum_{i=1}^{n} \int_{\Omega}\left|L_{i} P_{s} f\right|^{2} d \mu d s .
$$

For each $i=1, \ldots, n$, we again cut the integral into two parts with $M \geq 1$. The same commutation and contraction argument yield

$$
\int_{\left\{\left|L_{i} P_{s} f\right| \leq M\left\|L_{i} f\right\|_{1}\right\}}\left|L_{i} P_{s} f\right|^{2} d \mu \leq M\left\|L_{i} f\right\|_{1} \int_{\Omega}\left|L_{i} P_{s} f\right| d \mu \leq M\left\|L_{i} f\right\|_{1}^{2} .
$$

Therefore

$$
\operatorname{Var}_{\mu}\left(P_{t} f\right) \leq \frac{2 T}{1-e^{-T}}\left(M \sum_{i=1}^{n}\left\|L_{i} f\right\|_{1}^{2}+\frac{1}{T} \int_{t}^{t+T} \sum_{i=1}^{n} \int_{\left\{\left|L_{i} P_{s} f\right|>M\left\|L_{i} f\right\|_{1}\right\}}\left|L_{i} P_{s} f\right|^{2} d \mu d s\right) .
$$

Above the truncation level, for each $i=1, \ldots, n$, the same argument based on the Hölder and hypercontractivity inequalities yields

$$
\begin{aligned}
\int_{\left\{\left|L_{i} P_{s} f\right|>M\left\|L_{i} f\right\|_{1}\right\}}\left|L_{i} P_{s} f\right|^{2} d \mu & \leq \mu\left\{\left|L_{i} P_{s} f\right|>M\left\|L_{i} f\right\|_{1}\right\}^{\frac{1}{q}}\left\|L_{i} P_{s} f\right\|_{2 p}^{2} \\
& \leq\left\|L_{i} P_{s / 2} f\right\|_{2}^{2} \mu\left\{\left|L_{i} P_{s} f\right|>M\left\|L_{i} f\right\|_{1}\right\}^{\frac{1}{q(s)}} \\
& \leq \frac{1}{M^{\frac{1}{q(s)}}}\left\|L_{i} P_{s / 2} f\right\|_{2}^{2},
\end{aligned}
$$

with $q(s)=\frac{e^{\rho s}+1}{e^{\rho s}-1}$. Therefore,

$$
\int_{t}^{t+T} \sum_{i=1}^{n} \int_{\left\{\left|L_{i} P_{s} f\right|>M\left\|L_{i} f\right\|_{1}\right\}}\left|L_{i} P_{s} f\right|^{2} d \mu d s \leq \frac{1}{M^{\frac{1}{q(t)}}} \sum_{i=1}^{n} \int_{t}^{t+T}\left\|L_{i} P_{s / 2} f\right\|_{2}^{2} d s .
$$

Since

$$
\sum_{i=1}^{n} \int_{t}^{t+T}\left\|L_{i} P_{s / 2} f\right\|_{2}^{2} d s \leq \sum_{i=1}^{n} \int_{0}^{\infty}\left\|L_{i} P_{s / 2} f\right\|_{2}^{2} d s=\|f\|_{2}^{2},
$$


we thus get that

$$
\operatorname{Var}_{\mu}\left(P_{t} f\right) \leq \frac{2 \max (1, T)}{1-e^{-T}}\left(M\left(\sum_{i=1}^{n}\left\|L_{i} f\right\|_{1}^{2}\right)+\|f\|_{2}^{2} \frac{1}{M^{\frac{1}{q(t)}}}\right) .
$$

The theorem then follows as in the conclusion of the proof of Theorem 3.1, using moreover the fact that

$$
\inf _{T>0} \frac{4 \max (1, T)}{1-e^{-T}}=\frac{4}{1-e^{-1}} \leq 7 \text {. }
$$

\subsection{The case of more general Schreier graphs and non products examples}

This subsection briefly discusses further examples of interest, basically non-product models, for which the preceding approach may be developed similarly. The basic ingredients for such extension are the decomposition of the variance into directional derivatives and hypercontractivity.

Among discrete examples, the recent work of $[\mathrm{O}-\mathrm{W}]$ investigates the examples of general Schreier or Cayley graphs. In the context of Subsection 2.3, recall that the Dirichlet form $\mathcal{E}$ takes the form

$$
\mathcal{E}(f, f)=\frac{1}{2|S|} \sum_{x \in \Omega} \sum_{s \in S}\left[f\left(x^{s}\right)-f(x)\right]^{2} \mu(x)=\frac{1}{2|S|} \sum_{s \in S}\left\|D_{s} f\right\|_{L^{2}(\Omega)}^{2} .
$$

It is shown in $\mathrm{O}-\mathrm{W}$ that the commutation (14) holds with $\kappa=0$ (see also CE-L). Denote as usual by $\left(P_{t}\right)_{t \geq 0}$ the underlying semigroup attached to this Dirichlet form, and let $\lambda$ be the spectral gap constant and $\rho$ the logarithmic Sobolev constant. Noticing that there is a positive constant $C$ such that $\inf _{T>0} \frac{\max (1, T)}{1-e^{-\lambda T}} \leq \frac{C}{\lambda}$, we get the following theorem from the general scheme of proof developed in the preceding subsection.

Theorem 4.2. Let $\Omega$ be a Schreier or Cayley graph and $f: \Omega \rightarrow \mathbb{R}$. Then, for any $t \geq 0$,

$$
\mathcal{S}_{\eta}(f) \leq \frac{C}{\lambda}\left(\frac{1}{2|S|} \sum_{s \in \mathcal{S}} I_{s}(f)^{2}\right)^{\alpha(\eta)}\|f\|_{2}^{2-2 \alpha(\eta)},
$$

for some numerical constant $C>0$ and

$$
\alpha(\eta)=\frac{1-(1-\eta)^{\rho /(2 \lambda)}}{2} \geq \frac{\rho}{4 \lambda} \eta
$$

Thus, for a sequence of graphs $\left(\Omega_{n}\right)_{n \geq 0}$, we see from Theorem 4.2 that the original BenjaminiKalai-Schramm criterion holds whenever $\inf _{n \in \mathbb{N}} \frac{\rho_{n}}{\lambda_{n}}>0$, since in this case there exists an universal constant $c>0$ such that $\alpha_{n}(\eta) \geq c \eta$. Besides, Theorem 4.2 represents then a quantitative form similar to (2).

Explicit examples with known respective spectral gap and logarithmic Sobolev constants are given in $[\mathrm{O}-\mathrm{W}]$. Among them, we can cite the discrete tori $(\mathbb{Z} / m \mathbb{Z})^{n}, m \geq 2$. Then, given the product structure, both constants $\lambda_{n}$ and $\rho_{n}$ are of the same order, so that Theorem 4.2 provides a quantitative B-K-S relationship. The case of the Boolean cube $C_{n}$ with uniform measure can be seen as the Cayley graph $(\mathbb{Z} / 2 \mathbb{Z})^{n}$ generated by its canonical basis $S=\left(e_{i}\right)_{1 \leq n}$. Then the Dirichlet form is

$$
\mathcal{E}(f, f)=\frac{1}{2 n} \sum_{i=1}^{n}\left\|D_{i} f\right\|_{L^{2}\left(C_{n}\right)}^{2},
$$


so that the spectral gap constant and the hypercontractive constant are both equal to $\frac{2}{n}$ with this normalization. Rescaling by multiplying the Dirichlet form by $\frac{n}{2}$, we see that the above statement is in this case the one of $\mathrm{K}-\mathrm{K}$ and is therefore contained in Theorem 4.1 .

The main novelty with respect to the previous subsection is that it also covers two non product models of graphs, namely the symmetric group and the slices of the Boolean cube. In the case of the symmetric group $\mathfrak{S}_{n}$, recall its Cayley graph structure with the generating set given by the subgroup of the transpositions $\mathcal{T}_{n}$. The spectral gap $\lambda_{n}$ is equal to $\frac{2}{n-1}$, and the logarithmic Sobolev constant $\rho_{n}$ is greater than $\frac{a}{n \log n}$ for some $a>0$ (see [D-SC]). Hence, Theorem 4.2 does not improve upon the spectral gap inequality since $\frac{\rho_{n}}{\lambda_{n}}$ goes to 0 as $n$ goes to infinity. Notice that this conclusion also holds for the inequalities established in [CE-L] and [O-W] in the case of the symmetric group.

However, as pointed out in $[\mathrm{O}-\mathrm{W}]$, in the case of the the slices of the Boolean cube, both spectral gap and Sobolev logarithmic constants are of the same order. For $n \geq 1,1 \leq k<n$, the slices (of order $k$ ) of the Boolean cube are defined by $\left(\begin{array}{c}{[n]} \\ k\end{array}\right)=\left\{x \in\{0,1\}^{n}, \sum_{i=1}^{n} x_{i}=k\right\}$. The symmetric group is acting on $\left(\begin{array}{c}{[n]} \\ k\end{array}\right)$ by $x^{\sigma}=\left(x_{\sigma(i)}\right)_{1 \leq i \leq n}$, so that it has a Schreier graph structure. The generators are given by the transpositions $\tau_{i j}, 1 \leq i<j \leq n$, with $x^{\tau_{i j}}$ being obtained from $x$ by switching $i$ and $j$. Then, it is a result of Lee and Yau ([L-Y]) that the spectral gap constant is $\lambda=\frac{1}{n}$ and the logarithmic Sobolev constant $\rho$ satisfies $\rho^{-1} \sim n \log \frac{n^{2}}{k(n-k)}$. In particular when $\frac{k}{n}$ is bounded away from 0 and 1 , both constants are of the same order. We again rescale the Dirichlet form by multiplying by $n$ so that

$$
\mathcal{E}^{\prime}(f, f)=\frac{1}{n-1} \sum_{1 \leq i<j \leq n}\left\|D_{\tau_{i j}} f\right\|_{2}^{2} .
$$

Thus, the spectral gap constant associated to $\mathcal{E}^{\prime}$ is equal to 1 and the according Sobolev logarithmic constant $\rho^{\prime}$ satisfies $\rho^{\prime-1} \sim \log \frac{n^{2}}{k(n-k)}$. In particular, whenever $\frac{k}{n}$ is bounded away from 0 and 1 , $\rho^{\prime}$ is bounded away from 0 . Denoting by $\mathcal{S}_{\eta}^{s}=\mathcal{S}_{\eta}^{s_{k}}$ the noise stability in this context, Theorem 4.2 implies therefore that

$$
\mathcal{S}_{\eta}^{s} f \leq 7\left(\frac{1}{n-1} \sum_{1 \leq i<j \leq n}\left(I_{\tau_{i j}} f\right)^{2}\right)^{c \eta}\|f\|_{2}^{2-2 c \eta},
$$

for some positive constant $c$. We recall that $I_{\tau_{i j}} f$ is the influence of the transposition $\tau_{i j}$ on $f$. Therefore, the original [B-K-S] criterion holds over slices of the Boolean cube of order $k \in$ $\left[c^{\prime} n,\left(1-c^{\prime}\right) n\right]$, for each positive constant $c^{\prime}$. That is, if the sequence $\left(\frac{1}{n_{\ell}-1} \sum_{1 \leq i<j \leq n_{\ell}} I_{\tau_{i j}}^{2} f_{n_{\ell}}\right)_{n_{\ell} \geq 0}$ goes to 0 when $n_{\ell}$ goes to infinity, then the sequence of $\left(f_{n_{\ell}}\right)_{n_{\ell} \geq 0}$ is (asymptotically) noise sensitive. It is worth mentioning that a variant of this qualitative result over the slices has been recently established in [Fo, where the author uses this result in connection with the notion of exclusion sensitivity. We point out that (22) represents a quantitative version similar to the results of [K-K], K-M-S2]. One would be interesting to provide any application of this quantitative result.

\section{The case of the Euclidean spheres}

To conclude, we present the continuous model given by the Euclidean spheres in which the preceding scheme of proof apply. That is, it holds a decomposition (13) with commutation (14) as well as hypercontractivity (12) and spectral gap (8) (see [CE-L]). Although we could give a similar definition of noise stability, we will not do so as it has not a clear signification. We will express the results only in term of the heat semigroup associated to the spherical Laplacian. 
Let $\mathbb{S}^{n-1} \subset \mathbb{R}^{n}(n \geq 2)$, the $(n-1)$-dimensionnal Euclidean sphere equipped with its normalized surface measure $\mu$. Consider for $i, j \in\{1, \ldots, n\}, D_{i, j}=x_{j} \partial_{i}-x_{i} \partial_{j}$. The Dirichlet form associated with the spherical Laplacian

$$
\Delta=\frac{1}{2} \sum_{i, j=1}^{n} D_{i, j}^{2}
$$

takes the form

$$
\mathcal{E}(f, f)=\int_{\mathbb{S}^{n-1}} f(-\Delta f) d \mu=\frac{1}{2} \sum_{i, j=1}^{n} \int_{\mathbb{S}^{n-1}}\left(D_{i, j} f\right)^{2} d \mu .
$$

Since we clearly have $\Delta D_{i, j}=D_{i, j} \Delta$, (14) holds with $\kappa=0$. Consider again the heat semigroup $\left(P_{t} f\right)_{t \geq 0}=\left(e^{t \Delta} f\right)_{t \geq 0}$ generated by the Laplacian $\Delta$. It is known (see e.g. [Ba]) that the spectral gap constant and the logarithmic Sobolev constant are both equal to $n-1$. Our result can then be stated as follows.

Theorem 5.1. Let $f: \mathbb{S}^{n-1} \rightarrow \mathbb{R}$, where $n \geq 2$ be $C^{1}$-smooth and in $L^{2}(\mu)$. Then, for any $t \geq 0$,

$$
\operatorname{Var}_{\mu}\left(P_{t} f\right) \leq 7\left(\sum_{i, j=1}^{n}\left\|D_{i, j} f\right\|_{1}^{2}\right)^{\alpha(t)}\|f\|_{2}^{2-2 \alpha(t)},
$$

where

$$
\alpha(t)=\frac{1-e^{-(n-1) t}}{2} .
$$

Proof. The proof follows the scheme of the one of Theorem 3.1, with however the twist emphasized in the discrete framework of Section 4, i.e. restricting to a finite domain of integration in time $[0, T]$ for some $T \geq 0$ using (10) and replacing the logarithmic Sobolev constant by its value $n-1$. Then, we just notice that for every $\lambda \geq 1$

$$
\inf _{T>0} \frac{4 \max (1, T)}{1-e^{-\lambda T}}=\frac{4}{1-e^{-\lambda}} \leq 7 .
$$

Recall that the Poincaré inequality implies that $\operatorname{Var}_{\mu}\left(P_{t} f\right) \leq e^{-(n-1) t} \operatorname{Var}_{\mu}(f)$. Since the logarithmic Sobolev constant and the spectral gap constant are equal in this case, Theorem 5.1 provides some non-trivial bound on $\operatorname{Var}_{\mu}\left(P_{t} f\right)$ in specific ranges of $t$ (i.e. when $t$ of order $\frac{1}{n}$ ). To the best of our knowledge, this inequality is new. It would therefore be of interest to exhibit functions $\left(f_{n}\right)_{n \geq 0}$ over the Euclidean spheres such that the sequence $\left(\sum_{i, j=1}^{n}\left\|D_{i, j} f_{n}\right\|_{1}^{2}\right)_{n \geq 2}$ goes to 0 .

Acknowledgement. This work has been completed when I made my Ph.D in the University of Toulouse. I warmly thank my Ph.D advisor Michel Ledoux for introducing this problem to me, and for fruitful discussions. I also thank the anonymous referee for helpful comments in improving the exposition.

\section{References}

[Aal] C. Ané, S. Blachère, D. Chafaї, P. Fougères, I. Gentil, F. Malrieu, C. Roberto, G. Scheffer Sur les inégalités de Sobolev logarithmiques. Panoramas et Synthèses, Vol. 10 Soc. Math. de France (2000) 
[B-K-S] I. Benjamini, G. Kalai, O. Schramm Noise sensitivity of boolean functions and applications to percolation, Publ I.H.E.S 90, p. 5-43 (1999)

[Ba] D. BAKRY L'hypercontractivité et son utilisation en théorie des semigroupes., Ecole d'eté de Probabilités de Saint-Flour. Lecture Notes in Math. 1581, 1-114 1994. Springer (1994)

[B-E] D. BAKry, M. Emery Diffusions hypercontractives., Sminaire de Probabilits XIX, Lecture Notes in Math. Springer-Verlag, New York 1123 p.177-206 (1985)

[B-G-L] D. Bakry, I. Gentil, M. Ledoux. Analysis and geometry of Markov diffusion operators. Grundlehren der mathematischen Wissenschaften 348. Springer (2014).

[Be] W. Beckner Inequalities in Fourier analysis, Ann. of Math. 102(1), 159-182 1975. (1975)

[Bo] A. Bonami Étude des coefficients de Fourier des fonctions de $L^{p}(G)$, Annal. Inst. Fourier (Grenoble), 20 (2) 335-402 1971 (1970)

[CE-L] D. Cordero-Erausquin, M. Ledoux. Hypercontractives measures, Talagrand's inequality, and influences, Geometric Aspects of Functional Analysis, Springer, 2012, p. 169-189 (2011)

[D-SC] P. Diaconis, L. Saloff-Coste Logarithmic Sobolev inequalities for finite Markov chains, Ann. Appl. Prob. 6, 695-750 (1996)

[F] E. Friedgut Boolean functions with low average sensitivity depend on few coordinates. Combinatorica, 27-35 (1998)

[Fo] M.P. Fonsstöm A noise sensitivity theorem for Schreier graphs. Available online at http://arxiv.org/abs/1501.01828 (2015)

[K-K] N. Keller, G. Kindler Quantitative relationship between noise sensitivity and influences, Combinatorica 2013, Vol.33 : 45-71 (2013)

[K-M-S1] N. Keller, E. Mossel, A. Sen Geometric influences, Ann. of Probab. 2012, Vol.40 : $1135-1166(2012)$

[K-M-S2] N. Keller, E. Mossel, A. Sen Geometric influences II : Correlation inequalities and noise sentitivity, Ann. Inst. H. Poincaré, Vol.50, No4 : 1121 - 1139 (2014)

[K-S] G. Kalai, S. Safra Threshold phenomena and influence, Computational Complexity and Statistical Physics, Oxford Univ. Press, 25-60 (2006)

[G] L. Gross Logarithmic Sobolev inequalities, Amer. J. Math., 97: 1061 - 1083 (1975)

[G-S] C. Garban, J. E. Steif Lectures on Noise Sensitivity and Percolation, Cambridge University Press (2014)

[L] M. Ledoux The geometry of Markov diffusion generators., Ann. Fac. Sci., Toulouse IX, 305$366,(2000)$

[L-Y] T.Y. LeE, H.T. YAU Logarithmic Sobolev inequality for some models of random walks Ann. Of Probab. 1998; Vol.26 : 1855-1873 (1998)

[Ma] O. Mazet Classification des Semi-Groupes associés à une famille de polynômes orthogonaux Lecture Notes in Math. 1655, 40-53 1997. Springer, (1997) 
[N] E. Nelson The free Markov field. J. Funct. Anal. 12, 211-227 (1973)

[O-W] R. O'Donnell, K. Wimmer KKL, Kruskal-Katona, and monotone nets. 50th Annual IEEE Symposium on Foundations of Computer Science (FOCS 2009), 725734, IEEE Computer Soc., Los Alamitos, CA, (2009)

[T1] M. Talagrand On Russo's Approximate Zero-One Law, Ann. of Probab., 22 1576-1587 (1994)

[T2] M. TAlagrand How much increasing sets are positively correlated?, Combinatorica, 16 243$258(1996)$

[T3] M. Talagrand On Boundary and Influences, Combinatorica, 17 275-285 (1997)

RAPHAËL BOUYRIE,

Laboratoire d'Analyse de Mathématiques Appliqués, UMR 8050 du CNRS, Université Paris-

Est Marne-la-Vallée, 5 Bd Descartes, Champs-sur-Marne, 77454 Marne-la-Vallée Cedex, FRANCE

E-mail address: raphael.bouyrie@upem.fr 